\section{'Oral health crisis' looms}

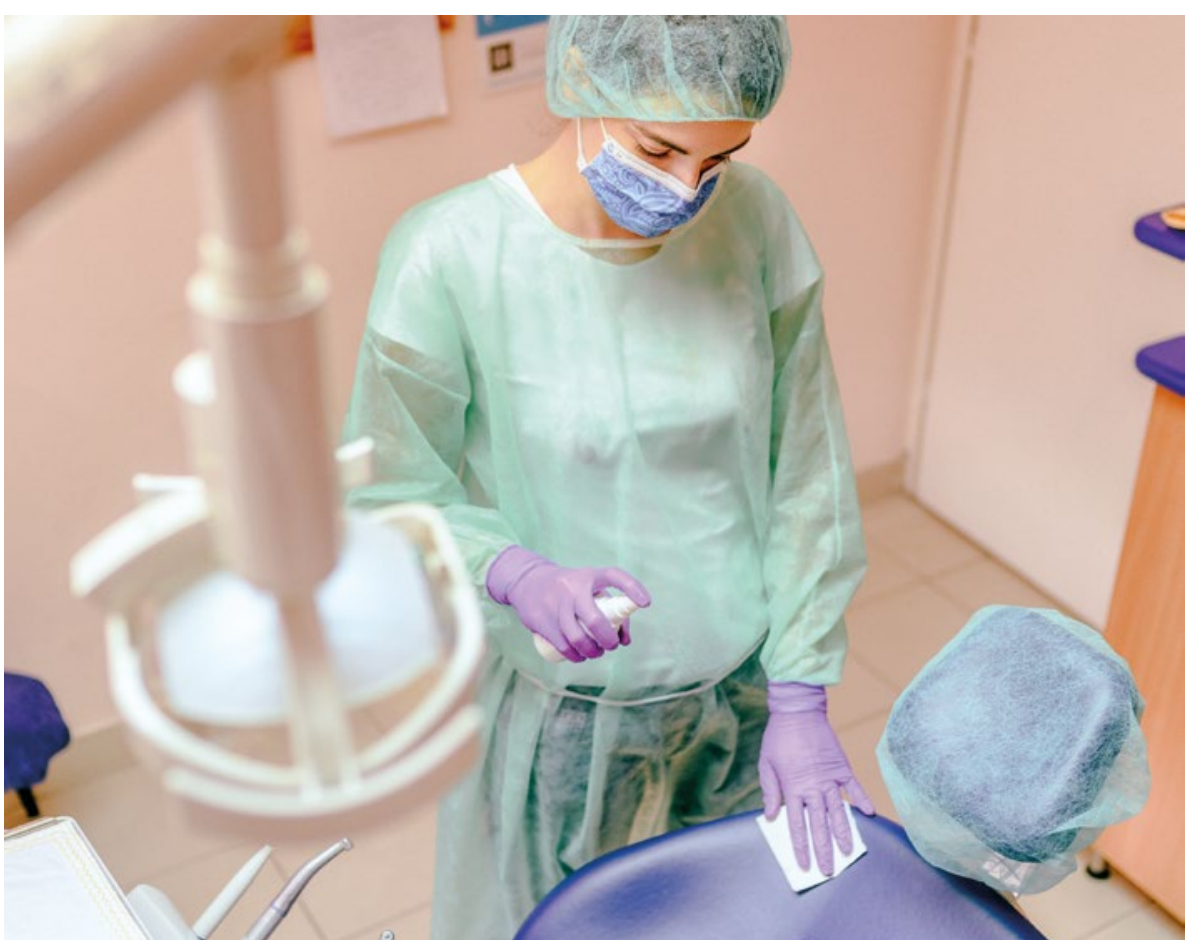

Professor Phil Taylor, Dean-Elect of the Royal College of Surgeons of Edinburgh's (RCSEd) Faculty of Dental Surgery, has expressed concern over the long-term oral health of the nation, one month after dental practices in England were given the green light to re-open.

RCSEd, which represents members all over the UK, says that while many dental practices are now seeing patients again, there is still a great deal of worry within the profession around the aftereffects of closures over recent months.

Professor Taylor said: 'We are now one month on from dental practices in England being allowed to re-open to treat non-emergency patients after the pandemic forced the vast majority of practices to close their doors.

'While it's fantastic that many practices are now back up and running, there are still significant hurdles to overcome. Dentists are operating at a much lower capacity than preCOVID due to stringent cleaning measures in between patients, and we know from a survey of our members that only half of those that are open feel they can provide the full range of fillings and hygiene appointments.

'Of course this improved protection is absolutely necessary in order to keep patients safe, but it does mean it will be a long time until dentists can effectively work through the backlog of patients which built up during the peak of the pandemic.
'There's still a lot of work to be done in convincing patients that it is safe to go to their dental practice for routine check-ups. Many may not feel comfortable in doing so for some time, but we want to emphasise that it shouldn't be avoided and is perfectly safe to do so. Unfortunately, some of those who do try to get an appointment may be unable to, due to practices' vastly reduced capacity.'

RCSEd's Faculty of Dental Surgery recently carried out a survey of its members, which showed that half of dentists believe they have patients who have suffered permanent harm as a result of practice closures, and $87 \%$ feel there is a backlog which will cause further long-term deterioration.

Professor Taylor continued: 'We could find ourselves in a situation where oral health in the UK is severely impacted for years to come. The concern is that with this longterm disruption caused by coronavirus, we will begin to see patients not being able to access regular and essential dental care which will inevitably lead to undiagnosed dental disease and particularly oral cancer.

'Since Dental Health Surveys were established in 1968, we've seen a considerable improvement in the nation's dental health. We do not want to reverse the trend and come to a place where dentures and missing teeth are the norm once again.'

\section{BSPD creates committee dedicated to research excellence}

A new committee has been created by the British Society of Paediatric Dentistry (BSPD) to have scrutiny over all aspects of the Society's work with academic and clinical governance components. The Quality and Improvement Research Committee (QIRC) will encourage all clinicians in the specialty to produce, disseminate and implement the highest quality evidence. It will coordinate national audits, prioritising ideas, reviewing protocols and allocating leads.

Other functions will include:

- Providing clinical expertise and guidance to BSPD

- Reviewing external guidelines and signposting to these

- Coordinating approaches for representation on external guidelines

- Advocating for academia, to ensure the best projects are funded

- Encouraging paediatric dentists to be research active and consider an academic career

- Having oversight of all requests to BSPD for questionnaires to be disseminated for membership feedback, making sure they are of value and scientifically valid

- Supporting BSPD's trainee research collaborative CONNECT

- Responsibility for the annual Clinical Effectiveness Bulletin.

Created at the behest of BSPD's Executive Committee, the group's formation has been led by Professor Paul Ashley, Senior Clinical Lecturer and Honorary Consultant at UCL, who will be the first Chair, supported by Dr Chris Vernazza, Senior Lecturer and Director of Research at the University of Newcastle's School for Dental Sciences.

Professor Ashley said: 'I am delighted to be asked to Chair this new and important committee for BSPD. It's our aim to drive the evidence base, support research and encourage more dentists to take a more active interest in research too.' 